\author{
H. J. Baek $\cdot$ S. W. Kang $\cdot$ Y. J. Lee $\cdot$ K. C. Shin \\ E. B. Lee $\cdot$ C. D. Yoo $\cdot$ Y. W. Song
}

\title{
Osteopenia in men with mild and severe ankylosing spondylitis
}

\begin{abstract}
We investigated the frequency and distribution of osteopenia according to the clinical severity in ankylosing spondylitis (AS). Bone mass was measured in men with mild $(n=45)$ and severe AS $(n=31)$ with dualenergy X-ray absorptiometry (DEXA). Definition of clinical severity was based on the Schober's test. Osteopenia was commonly detected $(48 \%$ in mild AS and $39 \%$ severe AS) and, in mild disease, more frequently observed at the lumbar spine than any of the proximal femur sites. In severe AS, however, the frequency of osteopenia at the femoral neck and Ward's triangle was as high as at the lumbar spine. Both bone mineral density and $T$-scores in severe disease were lower than in mild disease at the femur neck, Ward's triangle, and total proximal femur, but not in the lumbar spine. The progression of osteopenia may be reflected more reliably at proximal femur sites than at the lumbar spine.
\end{abstract}

Keywords Ankylosing spondylitis · Bone mineral density $\cdot$ Osteopenia $\cdot$ Osteoporosis

\section{Introduction}

Ankylosing spondylitis (AS) is a human leukocyte antigen (HLA)-B27-associated spondyloarthropathy that affects both sexes, but especially men. It is characterized by sacroiliitis and progressing spinal ankylosis with paravertebral ossification over years. It is known

H. J. Baek

Division of Rheumatology, Department of Internal Medicine, Gachon Medical School, Inchon, Korea

S. W. Kang $\cdot$ Y. J. Lee $\cdot$ K. C. Shin $\cdot$ E. B. Lee $\cdot$ C. D. Yoo Y. W. Song $(\square)$

Division of Rheumatology, Department of Internal Medicine, Seoul National University College of Medicine,

28 Yungun-dong, Chongno-gu, 110-744 Seoul, Korea

E-mail: ysong@snu.ac.kr

Tel.: + 82-2-7602234

Fax: $+82-2-7629662$ that patients with AS are prone to spinal fracture, which may be primarily attributed to spinal osteopenia $[1,2,3]$. Osteopenia is a well-known complication of AS, but its frequency, severity, anatomical distribution, and relation to the clinical severity of AS are controversial.

It is known that the dual photon absorptiometry values of lumbar bone mineral content were significantly diminished in male patients with radiographically less severe AS compared with controls but not in those with more severe disease [4]. In a dual-energy X-ray absorptiometry (DEXA) study, the mean bone mineral density (BMD) at the lumbar spine, in patients with radiographically severe AS, was higher than in those with mild AS but that this was not the case at the proximal femur [5]. Donnelly et al. reported that the DEXA Zscore at the proximal femur decreased in proportion to clinical disease severity (based on the Schober index), while the Z-score at the lumbar spine increased in the severe disease group vs the mild disease group [6]. Lee et al. showed that the DEXA Z-score at the lumbar spine was lower than that predicted for early disease subjects but not in the late disease group, while conversely the Z-score at the proximal femur was lower than predicted in late disease subjects but not in the early disease group [7].

We assessed bone mass of the lumbar spine and proximal femur in Korean male patients with AS using DEXA and analyzed the distribution of osteopenia according to the clinical severity of AS.

\section{Materials and methods}

We examined 76 consecutive men with AS who visited the rheumatology clinic in Seoul National University Hospital between March 1997 and August 1998. The patients fulfilled the modified New York criteria for AS [8]. Those with risk factors of osteoporosis, such as diabetes mellitus and steroid medication, were excluded. Careful history taking and physical examination, which included the modified Schober's test [9] and radiographs 
of the cervical, thoracic, and lumbar spine and pelvis were taken in each case.

Bone mass was measured at the lumbar spine (L2-4) and the proximal femur (femoral neck, Ward's triangle, greater trochanter, and total proximal femur) using DEXA (DPX and EXPERT, Lunar). Results are expressed as bone mineral density (BMD in $\mathrm{g} / \mathrm{cm}^{2}$ ) and $T$-scores. The $T$-score was defined as the standard deviation by which a patient's BMD differed from the mean BMD of normal Korean young controls, as provided by the Korean branch of the manufacturer. Osteopenia was defined by $T$-scores of $<-1$ and osteoporosis by $\leq-2.5$, which are a modification of WHO criteria [10]. Osteopenia included osteoporosis in our series. The functional class of patients was assessed according to the American College of Rheumatology 1991 revised criteria [11]. Clinically mild or severe disease was defined to be present when anteroposterior mobility of the lumbar spine was $\geq 5 \mathrm{~cm}$ or $<5 \mathrm{~cm}$, respectively. In this study, we compared the bone mass results and clinical features of cases with mild and severe disease.

\section{Results}

Clinical characteristics and the spinal X-ray abnormalities of patients are summarized in Table 1. Forty-five patients $(59.2 \%)$ were enrolled with mild disease and 31 $(40.8 \%)$ with severe disease. Mean ages at study entry and disease onset were higher in patients with severe disease than with mild disease $(32.7 \pm 7.1$ years vs $24.9 \pm 6.8$ years and $22.1 \pm 6.3$ years vs $16.2 \pm 5.3$ years, respectively) $(P<0.001$ by Student's $t$-test $)$. Functional class III/IV was more frequent in the severe disease group than in the mild disease group $(22.6 \%$ vs $2.2 \%)$ $(P=0.0067$ by Fisher's exact test). No difference between the two groups was found in the duration of disease or back symptoms. Syndesmophytes and abnormalities of facet joints on spinal radiography were more common in patients with severe disease than in those with mild disease $(64.5 \%$ vs $15.6 \%$ and $38.7 \%$ vs $4.4 \%$, respectively) $(P<0.001$ by chi-square test $)$. HLA B27 was found to be positive in all patients.

Frequency of osteopenia and osteoporosis

The frequencies of osteopenia and osteoporosis at the lumbar spine and total proximal femur were $47.7 \%$ and $4.5 \%$, respectively, in patients with mild disease, $38.7 \%$ and $12.9 \%$ in those with severe disease, and $43.4 \%$ and $7.9 \%$ in all patients (Table 2). Osteopenia was more frequently observed at the lumbar spine than any of the proximal femur sites in mild disease. In severe AS, however, its frequency at the femoral neck and Ward's triangle was as high as at the lumbar spine. Although the frequency of osteoporosis at the lumbar spine seemed to be higher than at the proximal femur in severe disease, this was not statistically significant. A difference in the frequencies of osteopenia between the mild and severe disease groups was observed only at Ward's triangle $(17.8 \%$ vs $51.6 \%)(P<0.01$ by chi-squared test $)$. Three cases $(3.9 \%)$ experienced lumbar vertebral fracture (all at L1), and all were included in the mild disease group.

Comparison of bone mass according to clinical severity

There was no difference in BMD or $T$-score at the lumbar spine between mild and severe AS (Table 3, Table 4). However, BMD (in $\mathrm{g} / \mathrm{cm}^{2}$ ) at the femur neck, Ward's triangle, and total proximal femur in severe disease was lower than in mild disease $(0.926 \pm 0.122$ vs $0.999 \pm 0.142, \quad 0.770 \pm 0.147 \quad$ vs $0.886 \pm 0.165, \quad$ and $0.956 \pm 0.117$ vs $1.036 \pm 0.142$, respectively $)(P<0.05$ by Student's $t$-test). $T$-scores at the same regions in severe disease were also lower than in mild disease $(-0.161 \pm 1.021 \quad$ vs $0.449 \pm 1.212,-0.842 \pm 1.132$ vs $0.047 \pm 1.265$, and $0.055 \pm 0.891$ vs $0.684 \pm 1.091$, respectively) $(P<0.05$ by Student's $t$-test $)$.

Table 1 Clinical characteristics and spinal X-ray abnormalities in patients with ankylosing spondylitis according to clinical severity ( $N S$ not significant)

\begin{tabular}{|c|c|c|c|c|}
\hline & Total $(n=76)$ & Mild disease $(n=45)$ & Severe disease $(n=31)$ & $P$ value $^{\mathrm{a}}$ \\
\hline Age at study (years) ${ }^{b}$ & $28.1 \pm 7.9$ & $24.9 \pm 6.8$ & $32.7 \pm 7.1$ & $<0.001^{*}$ \\
\hline Age at onset (years) ${ }^{b}$ & $18.6 \pm 6.4$ & $16.2 \pm 5.3$ & $22.1 \pm 6.3$ & $<0.001^{*}$ \\
\hline Disease duration (years) ${ }^{\mathrm{b}}$ & $9.4 \pm 5.1$ & $8.6 \pm 5.2$ & $10.6 \pm 4.9$ & NS \\
\hline Back symptom duration (years) ${ }^{\mathrm{b}}$ & $7.9 \pm 5.2$ & $7.1 \pm 5.7$ & $9.0 \pm 4.4$ & NS \\
\hline Functional class III/IV (\%) & $8(10.5)$ & $1(2.2)$ & 7 (22.6) & $0.0067^{* *}$ \\
\hline Modified Schober's test $(\mathrm{cm})^{\mathrm{b}}$ & $4.9 \pm 2.4$ & $6.6 \pm 1.1$ & $2.3 \pm 1.1$ & $<0.001^{*}$ \\
\hline Syndesmophyte $(\%)$ & $27(35.5)$ & $7(15.6)$ & $20(64.5)$ & $<0.001^{* * *}$ \\
\hline Facet joint disease $(\%)$ & $14(18.4)$ & $2(4.4 \%)$ & $12(38.7 \%)$ & $<0.001 * * *$ \\
\hline
\end{tabular}

${ }^{\mathrm{a} C o m p a r i n g ~ m i l d ~ a n d ~ s e v e r e ~ d i s e a s e ~ g r o u p s ~}$

${ }^{\mathrm{b}}$ Mean \pm standard deviation

*Student's $t$-test

**Fisher's exact test

***Chi-square test 
Table 2 Frequency of osteopenia/osteoporosis (\%) in patients with ankylosing spondylitis according to clinical severity (NS not significant)

\begin{tabular}{|c|c|c|c|c|}
\hline Site & Total $(n=76)$ & Mild disease $(n=45)$ & Severe disease $(n=31)$ & $P$ value $^{\mathrm{a}}$ \\
\hline Femoral neck & $11 / 1\left(14.5^{*} / 1.3\right)$ & $4 / 0(8.9 * / 0.0)$ & $7 / 1(22.6 / 3.2)$ & $\mathrm{NS} / \mathrm{NS}$ \\
\hline Trochanter & $6 / 0\left(7.9^{*} / 0.0^{* * *}\right)$ & $3 / 0(6.7 * / 0.0)$ & $3 / 0(9.7 * / 0.0)$ & NS/NS \\
\hline Total proximal femur & $6 / 0\left(7.9^{*} / 0.0^{* * *}\right)$ & $3 / 0(6.7 * / 0.0)$ & $3 / 0(9.7 * / 0.0)$ & $\mathrm{NS} / \mathrm{NS}$ \\
\hline L2-4 or total proximal femur & $33 / 6(43.4 / 7.9)$ & $21 / 2(47.7 / 4.5)$ & $12 / 4(38.7 / 12.9)$ & NS/NS \\
\hline
\end{tabular}

${ }^{\mathrm{a}} \mathrm{Chi}$-square test comparing the mild and severe disease groups

$* P<0.01$ by chi-square test compared with $\mathrm{L} 2-4$

** $P<0.05$ by chi-square test compared with L2-4

$* * * P<0.05$ by Fisher's exact test compared with L $2-4$

Table 3 Bone mineral density (BMD) in the patients with ankylosing spondylitis according to clinical severity (NS not significant)

\begin{tabular}{|c|c|c|c|c|}
\hline Site & Total $(n=76)$ & Mild disease $(n=45)$ & Severe disease $(n=31)$ & $P$ value $^{\mathrm{b}}$ \\
\hline L2-4 & $1.104 \pm 0.175$ & $1.100 \pm 0.167$ & $1.111 \pm 0.190$ & NS \\
\hline Femoral neck & $0.969 \pm 0.138$ & $0.999 \pm 0.142$ & $0.926 \pm 0.122$ & 0.023 \\
\hline Trochanter & $0.833 \pm 0.167$ & $0.852 \pm 0.132$ & $0.805 \pm 0.116$ & NS \\
\hline Total proximal femur & $1.004 \pm 0.137$ & $1.036 \pm 0.142$ & $0.956 \pm 0.117$ & 0.012 \\
\hline
\end{tabular}

$\mathrm{a} / \mathrm{cm}^{2}$, mean \pm standard deviation

'Student's $t$-test comparing mild and severe disease groups

Table $4 T$-score $^{\mathrm{a}}$ in patients with ankylosing spondylitis according to clinical severity (NS not significant)

\begin{tabular}{llll}
\hline Site & Total $(n=76)$ & Mild disease $(n=45)$ & Severe disease $(n=31)$ \\
\hline L2-4 & $-0.659 \pm 1.490$ & $-0.689 \pm 1.142$ & $-0.616 \pm 1.581$ \\
Femoral neck & $0.200 \pm 1.171^{*}$ & $0.449 \pm 1.212^{*}$ & $-0.161 \pm 1.021$ \\
Ward's triangle & $-0.316 \pm 1.282$ & $0.047 \pm 1.265^{* *}$ & $-0.842 \pm 1.132$ \\
Trochanter & $0.513 \pm 1.167^{*}$ & $0.682 \pm 1.218^{*}$ & $0.268 \pm 1.060^{* *}$ \\
Total proximal femur & $0.428 \pm 1.055^{*}$ & $0.684 \pm 1.091^{*}$ & $0.055 \pm 0.891^{* *}$ \\
\hline
\end{tabular}

${ }^{a}$ Standard deviation by which a patient's BMD differed from the mean BMD of normal Korean young controls, mean $\pm \mathrm{SD}$

bStudent's $t$-test comparing mild and severe disease groups

$* P<0.01$ by Student's $t$-test compared with L2-4

$* * P<0.05$ by Student's $t$-test compared with L2-4

Comparison of bone mass according to anatomical site

In mild disease, $T$-scores were lower at the lumbar spine than at any of the proximal femur sites $\left(P^{\prime}<0.05\right.$ by Student's $t$-test for all) (Table 4). In cases of severe disease, $T$-scores at the lumbar spine were lower than at the greater trochanter and total proximal femur $(-0.689 \pm 1.142$ vs $0.682 \pm 1.218$ and vs $0.684 \pm 1.091$, respectively) $(P<0.05$ by Student's $t$-test), but they were not different from those at the femoral neck and Ward's triangle. The $T$-scores were lowest at Ward's triangle.

\section{Discussion}

Osteopenia in AS is generally considered to be caused by the immobilization associated with spinal ankylosis and rigidity, and it is believed to be restricted to patients with advanced disease [12]. However, it has recently been reported that osteopenia frequently occurs in patients with early or mild AS who show little clinical or radio- graphic evidence of spinal disease [4, 5, 7, 13]. In agreement with these findings, we found that some of the patients with mild disease in our study $(47.7 \%)$ had osteopenia. This frequency was higher than in the severe disease group $(38.7 \%)$, although statistically insignificant.

The pathogenetic mechanisms of osteopenia are not clearly understood. In addition to immobilization, local or systemic cytokines that inhibit bone formation or augment bone resorption may be involved, since osteopenia was frequently observed even in patients with early or mild disease who were ambulatory and showed spinal mobility [14].

Bone mass measurements of the lumbar spine or proximal femur in AS patients depended upon the radiographic or clinical severity of the spinal disease and upon the measuring methods used $[4,5,6,7]$. In the present study, although the BMD and $T$-score at the lumbar spine were unaffected by clinical severity, those at the femoral neck, Ward's triangle, and total proximal femur were lower in the severe disease group than the 
mild disease group, which is comparable to the findings of Donnelly et al. and Lee et al. (Table 3, Table 4). Unlike previous reports [6,7], we did not find significantly increased bone mass at the lumbar spine in the severe disease group. This could have been due to the short disease duration of our severe disease group (mean duration $10.6 \pm 4.9$ years) as compared with these earlier reports.

Previous results $[4,7]$ and those of the present study show that bone mass at the lumbar spine, as measured by dual photon absorptiometry or DEXA, does not reflect the real progression of bone loss in AS patients. It is due to paravertebral syndesmophytes and abnormalities of the facet joints (fusion, sclerosis, etc.) in AS which influence bone mass measurement at lumbar spine. In our study, paravertebral syndesmophytes and abnormalities of the facet joints were found to be more frequent in severe AS patients than in mild AS patients (Table 1). Recent reports using quantitative computed tomography have revealed progressive bone loss in the central part of the vertebral body in spite of paravertebral calcification $[4,7]$.

Singh et al. showed that measurements of the femoral neck and Ward's triangle are more sensitive than lumbar measurements for the detection of osteopenia in patients with AS [15], but we believe that this might be applicable only to those with advanced AS. Our results suggest that the lumbar spine is a more sensitive site for the detection of osteopenia than the proximal femur in patients with mild AS (Table 2, Table 4). Our findings are supported by another study which found that bone mass measured at lumbar sites was lower than normative values in early AS, but that this was not apparent at the proximal femur [7]. Although osteopenia was often detected in cortical bone such as the femur neck (especially in severe AS patients), trabecular bone such as the lumbar vertebral body, or Ward's triangle was associated with more frequent and severe osteopenia (Table 2, Table 4). It appears that trabecular bone is more vulnerable to bone loss in AS.

Vertebral compression fractures due to osteoporosis are common but frequently unrecognized complications of AS and may contribute to the pathogenesis of spinal deformity and back pain [16]. In addition to compression fractures, transverse vertebral fractures also often occur in advanced AS patients. These observations reflect the fact that trabecular osteopenia and paravertebral calcification change the vertebral body into a tubular structure, like a long bone, and thus increase the likelihood of transverse vertebral fractures [1, 2, 3]. Ankylosed spines are likely to be fractured by minor trauma and result in significant morbidity, a high incidence of neurologic deficit, and even death in the case of cervical fractures $[1,2,3,17]$. The vertebral fracture rate in AS is reported to be $10.3 \%$ to $16.7 \%$ in Western countries $[6,16,18]$ and $40.9 \%$ in Turkey [19]. Our data suggest a fracture rate of $3.9 \%$, which is lower than previously reported. This may be attributed the younger age $(28.1 \pm 7.9$ years $)$ and shorter mean duration of disease $(9.4 \pm 5.1$ years) of our patients. Donnelly et al. demonstrated that vertebral fractures in AS occur with increasing age, disease duration, and severity of disease, and they further found that bone mass reduction in the lumbar spine or femoral neck is not a good predictor of the likelihood of fracture [6]. Interestingly, all the patients with vertebral fractures in our study belonged to the mild disease group. Moreover, it was recently reported that AS patients with mild disease also had a higher risk of fracture than the normal population [18].

In conclusion, osteopenia was frequently observed not only in patients with clinically severe AS but also in those with mild AS with little mobility limitation. Bone mineral density measurements, for the detection of osteopenia, were more sensitive at the lumbar spine than at any of the proximal femur sites in the mild disease group. In severe AS, however, the frequency of osteopenia at the femoral neck and Ward's triangle was as high as at the lumbar spine. Both BMD and $T$-scores in severe disease were lower than in mild disease at the femur neck, Ward's triangle, and total proximal femur but not in the lumbar spine. The progression of osteopenia in AS may be reflected more reliably at the proximal femur sites (except greater trochanter) than at the lumbar spine. It is probably due in part to progressing paravertebral calcification during the course of AS.

Acknowledgement This study was supported by a grant from Ministry of Science and Technology of Korea through the National Research Laboratory Program.

\section{References}

1. Hunter T, Dubo HI (1983) Spinal fractures complicating ankylosing spondylitis. A long-term follow up study. Arthritis Rheum 26:751-759

2. Murray GC, Persellin RH (1981) Cervical fracture complicating ankylosing spondylitis: a report of eight cases and review of the literature. Am J Med 70:1033-1041

3. Trent G, Armstrong GW, O'Neil J (1988) Thoracolumbar fractures in ankylosing spondylitis. High-risk injuries. Clin Orthop 227:61-66

4. Devogelaer JP, Maldague B, Malghem J, Nagant de Deuxchaisnes C (1992) Appendicular and vertebral bone mass in ankylosing spondylitis. A comparison of plain radiographs with single- and dual-photon absorptiometry and with quantitative computed tomography. Arthritis Rheum 35:1062-1067

5. Mullaji AB, Upadhyay SS, Ho EK (1994) Bone mineral density in ankylosing spondylitis. DEXA comparison of control subjects with mild and advanced cases. J Bone Joint Surg 76:660 665

6. Donnelly S, Doyle DV, Denton A, Rolfe I, McCloskey EV, Spector TD (1994) Bone mineral density and vertebral compression fracture rates in ankylosing spondylitis. Ann Rheum Dis $53: 117-121$

7. Lee YS, Schlotzhauer T, Ott SM, van Vollenhoven RF, Hunter J, Shapiro J, Marcus R, McGuire JL (1997) Skeletal status of men with early and late ankylosing spondylitis. Am J Med 103:233-241

8. van der Linden S, Valkenburg HA, Cats A (1984) Evaluation of diagnostic criteria for ankylosing spondylitis. A proposal for modification of the New York criteria. Arthritis Rheum $27: 361-368$ 
9. Macrae IF, Wright V (1969) Measurement of back movement. Ann Rheum Dis 28:584-589

10. Kanis JA, Melton LJ III, Christiansen C, Johnston CC, Khaltaev N (1994) The diagnosis of osteoporosis. J Bone Miner Res 9:1137-1141

11. Hochberg MC, Chang RW, Dwosh I, Lindsey S, Pincus T, Wolfe F (1992) The American College of Rheumatology 1991 revised criteria for the classification of global functional status in rheumatoid arthritis. Arthritis Rheum 35:498-502

12. Stewart AF, Adler M, Byers CM, Segre GV, Broadus AE (1982) Calcium homeostasis in immobilization: an example of resorptive hypercalciuria. N Engl J Med 306:1136-1140

13. Will R, Palmer R, Bhalla AK, Ring F, Calin A (1989) Osteoporosis in early ankylosing spondylitis: a primary pathological event? Lancet 2:1483-1485

14. Raisz LG (1988) Local and systemic factors in the pathogenesis of osteoporosis. N Engl J Med 318:818-828
15. Singh A, Bronson W, Walker SE, Allen SH (1995) Relative value of femoral and lumbar bone mineral density assessments in patients with ankylosing spondylitis. South Med J 88:939943

16. Ralston SH, Urquhart GD, Brzeski M, Sturrock RD (1990) Prevalence of vertebral compression fractures due to osteoporosis in ankylosing spondylitis. BMJ 300:563-565

17. Graham B, Van Peteghem PK (1989) Fractures of the spine in ankylosing spondylitis. Diagnosis, treatment, and complications. Spine 14:803-807

18. Mitra D, Elvins DM, Speden DJ, Collins AJ (2000) The prevalence of vertebral fractures in mild ankylosing spondylitis and their relationship to bone mineral density. Rheumatology 39:85-89

19. Sivri A, Kilinc S, Gokce-Kutsal Y, Ariyurek M (1996) Bone mineral density in ankylosing spondylitis. Clin Rheumatol $15: 51-54$ 